\title{
Real-Time Probing of Structural Dynamics by Interaction between Chromophores
}

\author{
Brogaard, Rasmus Y.; Møller, Klaus Braagaard; Sølling, Theis Ivan
}

Published in:

Journal of Physical Chemistry Part A: Molecules, Spectroscopy, Kinetics, Environment and General Theory

Link to article, DOI:

10.1021/jp2072588

Publication date:

2011

Document Version

Publisher's PDF, also known as Version of record

Link back to DTU Orbit

Citation (APA):

Brogaard, R. Y., Møller, K. B., \& Sølling, T. I. (2011). Real-Time Probing of Structural Dynamics by Interaction between Chromophores. Journal of Physical Chemistry Part A: Molecules, Spectroscopy, Kinetics, Environment and General Theory, 115, 12120-12125. https://doi.org/10.1021/jp2072588

\section{General rights}

Copyright and moral rights for the publications made accessible in the public portal are retained by the authors and/or other copyright owners and it is a condition of accessing publications that users recognise and abide by the legal requirements associated with these rights.

- Users may download and print one copy of any publication from the public portal for the purpose of private study or research.

- You may not further distribute the material or use it for any profit-making activity or commercial gain

- You may freely distribute the URL identifying the publication in the public portal 


\title{
Real-Time Probing of Structural Dynamics by Interaction between Chromophores
}

\author{
Rasmus Y. Brogaard, ${ }^{\dagger}$ Klaus B. Møller, ${ }^{\ddagger}$ and Theis I. Sølling, ${ }^{*}$ \\ ${ }^{\dagger}$ Department of Chemistry, University of Copenhagen, Copenhagen, Denmark \\ ${ }^{\ddagger}$ Department of Chemistry, Building 207, Technical University of Denmark, Kgs. Lyngby, Denmark
}

ABSTRACT: We present an investigation of structural dynamics in excited-state cations probed in real-time by femtosecond timeresolved ion photofragmentation spectroscopy. From photoelectron spectroscopy data on 1,3-dibromopropane we conclude that the pump pulse ionizes the molecule, populating an excited electronic state of the radical cation. In this state a coherent torsional vibration of the bromomethylene groups with a period of $700 \mathrm{fs}$ is started and probed by photoinduced fragmentation of the molecular cation. The vibrational coherence dephases with the decay of the excited state to the ground state of the cation in $1.6 \mathrm{ps}$.

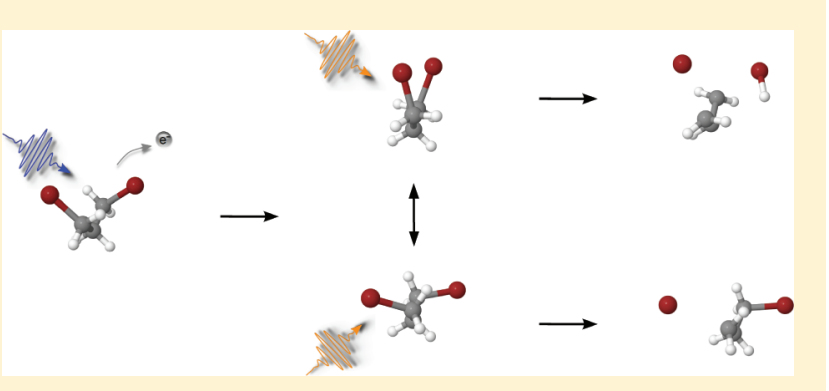
The real-time probing of the excited-state dynamics is made possible by exploiting the interaction between the two bromine chromophores and its dependence on molecular conformation. This experiment therefore illustrates the applicability of the concept of probing ultrafast molecular dynamics using the intramolecular interaction between two chromophores.

\section{INTRODUCTION}

By now, the amount of papers on gas phase femtosecond timeresolved studies of molecular dynamics of neutrals and anions is enormous. On the contrary the work on ultrafast dynamics of cations in the gas phase is rather limited. ${ }^{1-10}$ In these experiments the pump pulse generates the cation by multiphoton ionization and absorption of the probe pulse induces increased fragmentation of the molecular ion, a scheme referred to as timeresolved ion photofragmentation (TRPF) by Ho et al. ${ }^{1}$ When the wave packet passes a region of the potential energy surface (PES) of the electronic state of the cation where the absorption cross section of the probe pulse is increased, sometimes called a "dynamic resonance", 4,6 absorption will lead to increased fragmentation of the molecular ion, either by direct means through dissociative states or in a statistical process as a consequence of excess internal energy. The position of these dynamic resonances depends on the shape of the PESs and the probe wavelength. By changing the latter different parts of the wavepacket trajectories can be probed. As such, TRPF experiments bear a large resemblance to transient absorption experiments.

Excited-state dynamics in molecular cations is a promising research field, considering the myriad of examples of nonadiabatic effects giving rise to exciting ultrafast phenomena that have been observed in excited states of neutral molecules. In this paper we report on real-time probing of structural changes in an excited state of a molecular cation, taking place in the multichromophoric radical cation of 1,3-dibromopropane (DBP). Ultrafast reactivity is often associated with a particular conformation and it was appreciated already early on that the role of conformational dynamics in excited-state chemistry is quite different from that in ground-state chemistry. ${ }^{11}$ Multichromophoric molecules (neutral as well as ionic) are particularly well suited for investigations of structural dynamics, because the interaction between the chromophores can be used to probe the molecular conformation. The probing of conformational dynamics in DNA is a famous example (see, e.g., ref 12 and references therein). As it turns out, compounds containing multiple chromophores are also well suited for studying excited-state cation dynamics. This is most clearly appreciated by employing the Koopmans picture (ref 13 and references therein), in which the ionization process involves a single active electron and leaves the electronic configuration of the core unchanged. Consider the case in which the radical cation is created by the pump pulse in a resonance enhanced multiphoton ionization (REMPI) process, in which the ionization occurs through an intermediate excited state in the neutral molecule. If this state involves an excitation of an electron from the HOMO, it will exhibit a (more or less pronounced) propensity toward populating the electronic ground state, $\mathrm{D}_{0}$, of the cation in the ionization process. This relation has been referred to as an ionization correlation between the neutral excited state and the cation state. ${ }^{13-16}$ On the other hand, if the intermediate state is described by an excitation involving a chromophore that is not associated with the HOMO, it will exhibit an ionization correlation to an excited state, $\mathrm{D}_{x}$, of the cation. These two cases are illustrated in Figure 1. Formation of excited-state ions by REMPI involving intermediate excitations of different chromophores has been observed experimentally, e.g., in experiments on $\mathrm{N}, \mathrm{N}$-dimethyl-2-phenylethylamine. ${ }^{17}$ At a wavelength of $400 \mathrm{~nm}$ the amine moeity is ionized, forming the cation in the ground state. With a $267 \mathrm{~nm}$ pulse, an electron is removed from the phenyl ring, forming the

Received: July 29, 2011

Revised: September 28, 2011

Published: September 29, 2011 
$\mathrm{D}_{0}$
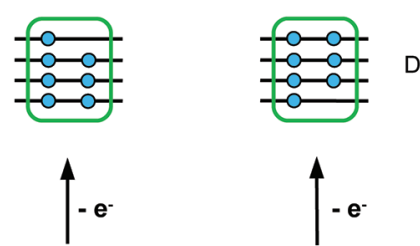

$\mathrm{S}_{\mathrm{a}}$
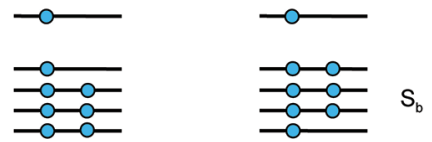

Figure 1. Illustration of ionization correlations for two excited states $S_{a}$ and $S_{b}$. The correlations can be rationalized using the Koopmans picture of photoionization that involves a single active electron and leaves the electronic configuration of the core unchanged.

cation in an excited electronic state, because the ring is the chromophore involved in the intermediate state of the REMPI process. ${ }^{17}$

As will be elaborated below, interaction between the two bromine atoms in DBP lifts the degeneracy of the lone pairs. This means that they can be considered different chromophores, making DBP well-suited for investigations of excited-state cation dynamics. Using TRPF spectroscopy, we aim to understand in detail the dynamics of the radical cation and unravel which photofragmentation channels are opened by absorption of the probe pulse. Furthermore, we supplement the TRPF experiments with time-resolved photoelectron spectroscopy (TRPES) experiments. The TRPES data will reveal any contribution of neutral excitedstate dynamics to the ion signals and will be used as a proof that we are indeed probing cation dynamics.

\section{EXPERIMENTAL SECTION}

1,3-Dibromopropane (99\%) was purchased from Sigma-Aldrich and used without further purification.

The TRPF and TRPES experiments were carried out using a femtosecond pulsed laser system and the Copenhagen molecular beam mass and photoelectron spectrometer, which has been described previously. ${ }^{18,19}$ The laser system consists of a chirped pulse amplifier (Spitfire, Spectra Physics) seeded by a femtosecond oscillator (Tsunami, Spectra Physics). The amplifier delivers $800 \mathrm{~nm}$ pulses of $100 \mathrm{fs}$ duration ( $\mathrm{fwhm}$ ) and $1 \mathrm{~mJ}$ energy at $1 \mathrm{kHz}$ repetition rate. The output was divided by a 50:50 beam splitter into two parts pumping an OPA (TOPAS-C, Light Conversion) and a higher harmonics generation setup, respectively. The latter setup delivered the $267 \mathrm{~nm}$ (third harmonic of the fundamental) pulses used as pump pulses with an energy of $5-7 \mu \mathrm{J}$. The $620 \mathrm{~nm}$ probe pulses, with an energy of $11-12 \mu \mathrm{J}$, were generated by sum frequency mixing the OPA output (signal) and the $800 \mathrm{~nm}$ fundamental. The polarizations of the pump and probe pulse were kept at magic angle and the time delay between the pulses was controlled by a motorized translation stage. The stage and the data acquisition were controlled by a LabVIEW routine. The in situ one pump + two probe photon $\left(\left[1,2^{\prime}\right]\right)$ cross correlation between the pulses was determined to be $165 \mathrm{fs}$ using dimethylisopropylamine. Assuming identical durations of the pump and probe pulse this value was reduced by a factor of $3^{1 / 2} /$ 2 to 140 fs to reflect the $\left[3,1^{\prime}\right]$ scheme that probes the ion dynamics in the experiment.

The photoelectron spectrum was calibrated at several wavelengths using the well-known Rydberg peaks of $\mathrm{N}, \mathrm{N}$-dimethyl- $g, g-C_{2}$

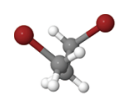

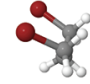

$g, g-C_{s}$

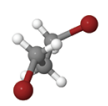

a,g- $C_{1}$

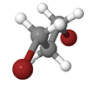

$\mathrm{a}, \mathrm{a}-\mathrm{C}_{2 \mathrm{v}}$
Figure 2. Four conformers of 1,3-dibromopropane.

isopropylamine. ${ }^{20}$ Because we often observe peaks from autoionizing superexcited states below $0.2 \mathrm{eV}$, all photoelectron spectra have been cut below this threshold.

In the interaction region a continuous molecular beam was intersected at right angle by the incoming laser pulses. The beam was generated by supersonic expansion of sample vapor $\left(23^{\circ} \mathrm{C}\right)$ seeded in 0.3 bar He. The expansion nozzle was heated to $70-80{ }^{\circ} \mathrm{C}$ to prevent condensation.

All calculations were carried out using the Gaussian03 suite of programs. ${ }^{21}$ Heats of formation and barrier heights for the ground-state cation of 1,3-dibromopropane were calculated at the G3//B3LYP level of theory. ${ }^{22}$

\section{RESULTS AND DISCUSSION}

Ground-State Structural Aspects. Because structural dynamics is a major part of this work, we will start by considering the four conformers of DBP shown in Figure 2. From electron diffraction studies it was determined that in a gas phase sample of DBP at $65{ }^{\circ} \mathrm{C}$ (a temperature close to our experimental conditions) there is $67 \% \mathrm{~g}, \mathrm{~g}-C_{2}, 30 \% \mathrm{a}, \mathrm{g}-C_{1}$, and $3 \% \mathrm{a}, \mathrm{a}-C_{2 v}$ (the $\mathrm{g}, \mathrm{g}-C_{s}$ conformer was not present in detectable amounts), ${ }^{23}$ reflecting that there is a stabilizing interaction between the bromine atoms. In competition with steric hindrance. This interaction is greatly enhanced in the ground-state radical cation, in which the gauche-gauche conformers dominate. ${ }^{24}$ Because it was not possible to determine the distribution among the g,g- $C_{2}$ and the g,g$C_{s}$ conformer from the experimental results, ${ }^{24}$ we have calculated heats of formation of the conformers of the cation, which predict a stabilization of the g,g- $C_{s}$ conformer by $18 \mathrm{~kJ} / \mathrm{mol}$ compared to that of the g,g- $C_{2}$ conformer. This shows that at equilibrium the $\mathrm{g}, \mathrm{g}-C_{s}$ will be the only conformer present in a sample of groundstate radical cations of DBP in the gas phase. Ab initio calculations reflect that ionization increases the interaction between the bromine atoms in the optimized geometry of g,g- $C_{2}$; the dihedral angle $\mathrm{D}(\mathrm{BrCCC})$ is $58^{\circ}$ in $\mathrm{S}_{0}$, whereas it is only $30^{\circ}$ in $\mathrm{D}_{0}{ }^{25}$ This means that the removal of an electron can be expected to induce a conformational change in the resulting cation along a torsional vibration of the bromomethylene groups. In fact, the results discussed below do indeed show that this structural change is induced by ionization with the pump pulse and followed through induced fragmentation of the cation by the probe pulse.

Photoelectron Spectroscopy. Figure 3 shows the photoelectron spectrum obtained from the pump pulse $(267 \mathrm{~nm})$ only. When considering that the total energy of three photons of $267 \mathrm{~nm}$ is $13.9 \mathrm{eV}$ and that the vertical ionization potential of DBP is $10.1 \mathrm{eV}^{26}$ this should leave a maximum of $13.9-10.1=$ $3.8 \mathrm{eV}$ as kinetic energy to the photoelectron. Closer inspection reveals a very weak onset of the photoelectron signal around 3.3 $\mathrm{eV}$, fitting reasonably well with the value of $3.8 \mathrm{eV}$ considering the background of electrons generated by higher-order processes involving a larger number of photons. The spectrum is unstructured and extends over a wide range of energies, indicating that nonresonant ionization dominates. There is a maximum at 0.4 $\mathrm{eV}$, though, which we assign to ionization of a $(\mathrm{n}, 5 \mathrm{~d})$ Rydberg 


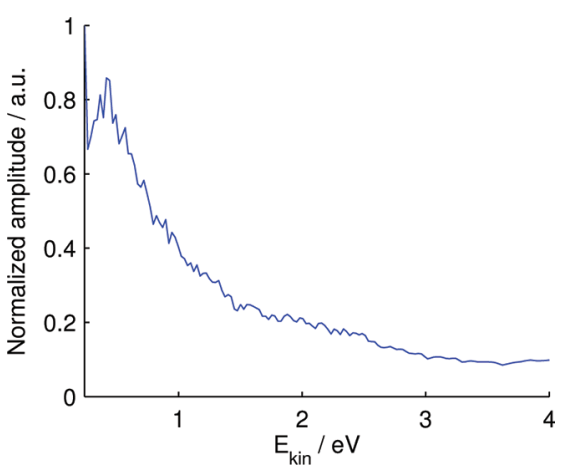

Figure 3. Photoelectrum spectrum of 1,3-dibromopropane obtained from ionization with $267 \mathrm{~nm}$ pulses only.

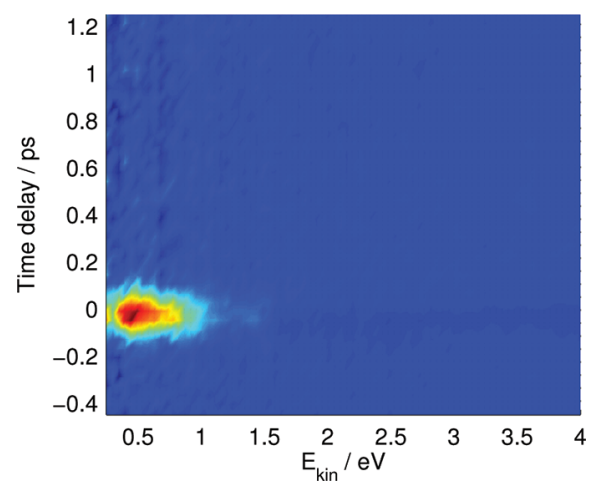

Figure 4. Time-resolved photoelectron spectrum (after subtraction of one-color signal) of 1,3-dibromopropane excited with $267 \mathrm{~nm}$ pulses and probed with $620 \mathrm{~nm}$ pulses.

state excited resonantly during a three-photon REMPI process, because the energy of two $267 \mathrm{~nm}$ photons matches the calculated excitation energy region of the (n,5d) Rydberg manifolds. ${ }^{25}$ Considering the remarkable energy difference between this peak and the onset of the photoelectron spectrum, $3.3-0.4=2.9 \mathrm{eV}$, we find it unlikely that the excess energy is deposited only in vibrational energy but take it to indicate that the Rydberg state is ionized to an excited electronic state of the cation. The assignment of the particular state is done per the discussions below.

The time-resolved photoelectrum spectrum of DBP after subtraction of one-color signal is shown in Figure 4. As was the case for the one-color pump signal, the spectrum is broad and unstructured. Consider first a reversed pump-probe scheme in which three photons of the probe pulse excite a repulsive $\left(\mathrm{n}, \sigma^{*}\right)$ state situated $6 \mathrm{eV}$ above the ground state $\mathrm{e}^{25}$ that is ionized by the pump pulse. This process is also expected to result in a broad spectrum, due to the repulsive nature of the excited state. Thus, we do not find that further analysis aiming at disentangling the pump-probe from the probe-pump signal is justified. However, the most important result from the TRPES experiments is that the time-resolved signal and thus any dynamics involving neutral species are cross-correlation limited, which supports that the dynamics observed at longer delay times in the ion transients discussed below is purely cation dynamics.

Mass Spectrometry. The analysis of the time-resolved mass spectrometry data will focus on the parent and fragment ions shown in Figure 5. The temporal evolution of these ion currents, all normalized to a maximum amplitude of one, is shown in

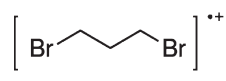

$200 / 202 / 204 \mathrm{amu}$

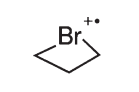

$121 / 123 \mathrm{amu}$
$\widehat{\mathrm{CH}_{2}^{+}}$

$41 \mathrm{amu}$
Figure 5. Molecular and fragment ions of 1,3-dibromopropane corresponding to the signals in the time-resolved mass spectrum that the data analysis of the TRPF results focuses on.

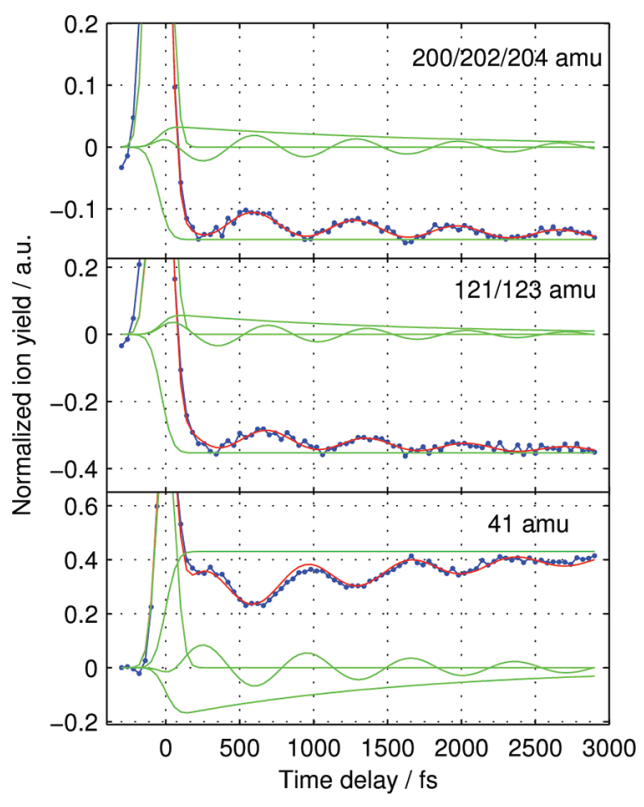

Figure 6. Temporal evolution of selected signals (all normalized to a maximum amplitude of one) in the time-resolved mass spectrum of 1,3dibromopropane excited with $267 \mathrm{~nm}$ and probed with $620 \mathrm{~nm}$ pulses. Also shown in the figure are the components of the function used to fit the signals.

Figure 6. Also shown in the figure are the components of the functions used to fit the signals. Besides the initial cross correlation limited signal and a constant offset, the transients are composed of two components; an exponential rise/decay and a damped oscillation. The period of the oscillation is $700 \mathrm{fs}$. The fits were made such that the damping of the oscillation is the same as the exponential rise/decay time, which is $1.6 \mathrm{ps}$. We observe that there is a phase shift of $\pi$ of the oscillation in the parent ion (202 amu) transient as compared to the oscillation in the $41 \mathrm{amu}$ transient. As can be seen, the amplitude of the oscillatory signal is rather weak, which fits well with the TRPES results that indicate a low contribution from resonant processes giving rise to the dynamics.

In the following the two components of the experimental signals are interpreted, starting with the oscillatory part. The latter is a clear sign that the ionization by the pump pulse creates a wavepacket in the cation state that spans a number of vibrational levels of a low frequency vibration, thereby starting a coherent motion along that coordinate. The vibrational period corresponds to a frequency of $48 \mathrm{~cm}^{-1}$. This value is remarkably close to the value of $45 \mathrm{~cm}^{-1}$ that was calculated for a torsional vibration of the bromomethylene groups in an $(n, 5 p)$ Rydberg state. ${ }^{25}$ The nuclear motion along this coordinate, from here on called the bromotorsion, is illustrated in Figure 7. Note that, because of the ionic nature of Rydberg states, it is to be expected that there is an electronic state of the cation with an associated PES that is 


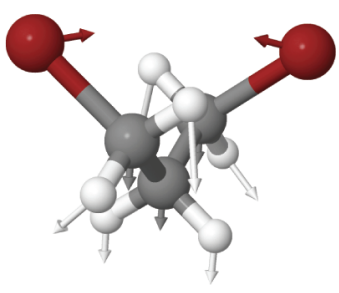

Figure 7. Illustration of the nuclear motion in the bromotorsion vibration in the $\mathrm{g}, \mathrm{g}-\mathrm{C}_{2}$ conformer of 1,3-dibromopropane.

practically parallel to that of the Rydberg state along the bromotorsion coordinate. In other words, it is very likely that the wave packet motion along the bromotorsion coordinate that was shown to take place in one of the $(n, 5 p)$ Rydberg states by quantum dynamics simulations ${ }^{27}$ can take place in an (excited) electronic state of the cation as well. High level ab initio calculations do indeed show that the topology of the PES of the $\mathrm{D}_{3}$ state along the bromotorsion coordinate is almost identical to that of the Rydberg state. ${ }^{25}$ Thus, we assign the observed oscillatory behavior of the signal to the bromotorsion vibration initiated in the $\mathrm{D}_{3}$ excited state of the cation. This is explained by the involvement of the Rydberg state as an intermediate state that, due to the strong ionization correlations of Rydberg states, preferentially populates $\mathrm{D}_{3}$ in the ionization step of the REMPI process. The assignment is further supported when considering the photoelectron spectroscopy results that indicate the population of an excited state of the ion. Second, we have calculated the vibrational frequency (B3LYP/6-31+G(d)) of the bromotorsion vibration in the $\mathrm{D}_{0}$ state to be $110 \mathrm{~cm}^{-1}$, corresponding to a vibrational period of $300 \mathrm{fs}$, making it seem unlikely that the vibration takes place in $\mathrm{D}_{0}$. As mentioned above, the reason that this motion can be monitored in the experiment is the presence of a dynamic resonance along the vibrational coordinate. The resonance induces an increased fragmentation of the ion due to absorption of one or more photons of the probe pulse. The position of the resonance is revealed from the phase of the oscillation in the transients; the maximal fragmentation is seen after one-half of the vibrational period. Thus, the resonance is situated close to the outer turning point of the vibrational coordinate. In this region of the PES, the bromine atoms are closer to each other $\left(\mathrm{D}(\mathrm{BrCCC}) \sim 30^{\circ}\right)$ and interact in a way that enhances the probability of absorbing the probe photon. This is sketched in Figure 8. A similar phenomenon was observed in a TRPF experiment on the radical cation of $\mathrm{CH}_{2} \mathrm{I}_{2}$ in which a scissoring vibration of the iodine atoms could be monitored due to the fact that concerted elimination of $\mathrm{I}_{2}{ }^{+}$induced by the probe pulse preferentially occurred when the iodine atoms were close. ${ }^{5}$

Turning to the second component in the fits of the transients, the decay/rise, we point out that because the damping time of the oscillations can be fitted to the same value as the decay/rise components, they are most likely related. We explain this by assigning the decay time to internal conversion from the initially excited electronic state, $D_{3}$, to the ground state, $D_{0}$. The internal conversion depopulates the $\mathrm{D}_{3}$ state, thereby damping the oscillations in the signal. In the $\mathrm{D}_{0}$ state the minimum along the bromotorsion coordinate is located at $\mathrm{D}(\mathrm{BrCCC})=30^{\circ}$, as opposed to $\sim 45^{\circ}$ in $\mathrm{D}_{3}$. Hence, the internal conversion $\mathrm{D}_{3} \rightarrow \mathrm{D}_{0}$ will bring the bromine atoms closer together. This enhances the absorption cross section of the probe pulse and thereby fragmentation, which is why the internal conversion is seen as a rise in $41 \mathrm{amu}$ and a corresponding decay in 202/121 amu. It is
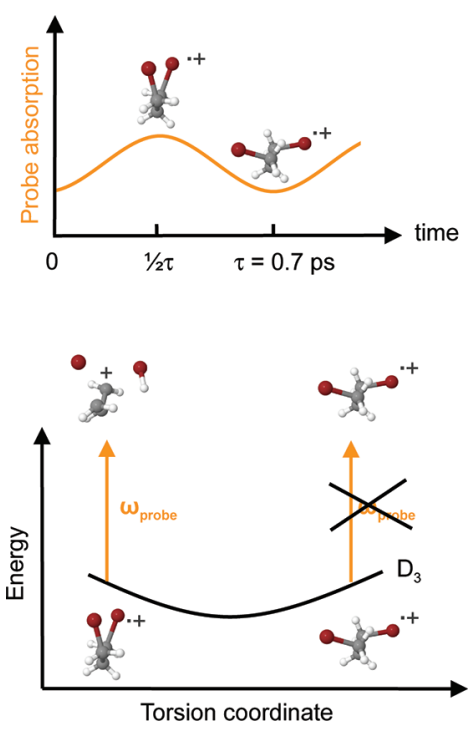

Figure 8. Sketch illustrating how the coherent vibration in the radical cation of 1,3-dibromopropane is followed in the TRPF experiment. The absorption cross section of the probe photon is modulated along the bromotorsion coordinate; when the bromine atoms are close, the cross section is high, leading to an increased fragmentation of the parent ion $(202 \mathrm{amu})$ to the fragment $(41 \mathrm{amu})$. When the bromine atoms are further apart, the cross section, and thereby fragmentation, is lower.

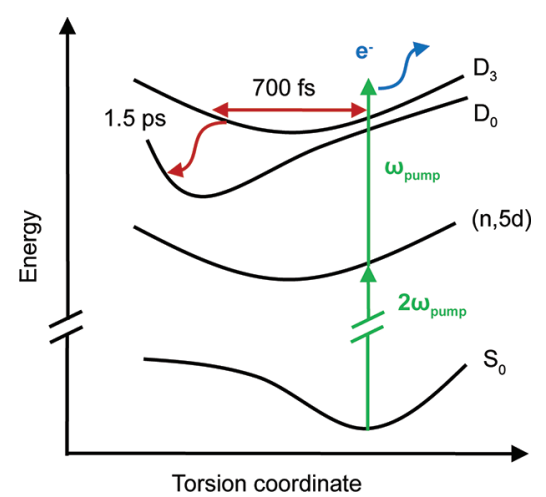

Figure 9. Sketch illustrating the molecular dynamics initiated in the $\mathrm{D}_{3}$ excited state of the radical cation when 1,3-dibromopropane is ionized by the pump pulse $(267 \mathrm{~nm})$ through an intermediate (n,5d) Rydberg state.

stressed that the internal conversion to $\mathrm{D}_{0}$ takes place in the g,g$\mathrm{C}_{2}$ conformation. This can be appreciated by considering that the calculated barrier for the conformational change from g,g- $C_{2}$ to $g$, $\mathrm{g}-\mathrm{C}_{s}$ in $\mathrm{D}_{0}$ is $54 \mathrm{~kJ} / \mathrm{mol}$ and that the corresponding barrier in $\mathrm{D}_{3}$ is expected to be similar. This means that, when the $D_{3}$ state is excited, the barrier for the conformational change cannot be crossed due to insufficient energy. Thus, the internal conversion from $\mathrm{D}_{3}$ to $\mathrm{D}_{0}$ has to take place in geometries close to g,g- $C_{2}$. Following the internal conversion, it is likely that the conformational change from g,g- $C_{s}$ to g,g- $C_{2}$ takes place, but it will compete with the cleavage of the $\mathrm{C}-\mathrm{Br}$ bond and both processes will be statistical in nature.

Unifying Picture. Summing up the above, our interpretation of the results is as follows: the pump ionizes the molecule populating the $\mathrm{D}_{3}$ excited state of the cation and initiating a coherent torsional vibration of the bromomethylene groups. The 
dephasing of the coherent motion is a result of the $\mathrm{D}_{3}$-state population decaying by internal conversion to the $D_{0}$ state. This scenario is sketched in Figure 9. We note that our experimental data are very similar to those obtained by Kötting et al. on the same molecule, ${ }^{25}$ and it therefore seems likely that what they observed was indeed dynamics in the cation. It is important to keep in mind that whereas we used a probing wavelength that is almost identical to what Kötting et al. used (620 and $615 \mathrm{~nm}$, respectively), the wavelengths of the pump pulses are quite different ( 267 and $308 \mathrm{~nm}$, respectively). What both experiments have in common, though, is that the intermediate state involved in the REMPI process of the pump step is located within a Rydberg manifold; the $(n, 5 p)$ in their case and the $(n, 5 d)$ in our case. Because the PESs of the lowest excited states of the cation have very different topologies along the bromotorsion coordinate depending on which lone pair the electron was removed from, ${ }^{25}$ the fact that the observed time scales are close to identical seems to imply that the $\mathrm{D}_{3}$ state is populated in both experiments. This further implies that the intermediate Rydberg states involved in the REMPI process, have the same configuration of the ionic core, i.e., involves an excitation of an electron from the same lone pair, the HOMO-3 orbital. This might seem strangely coincidental, considering that the intermediate states are located within Rydberg manifolds of $4 \times 3=12(n, 5 p)$ and $4 \times 5=20$ $(n, 5 d)$ energetically closely lying states, respectively. We believe that it is a consequence of Franck-Condon overlap; as shown in calculations by Kötting et al. the Rydberg PESs along the bromotorsion coordinate are almost identical to the corresponding (excited) states of the cation. ${ }^{25}$ Thus, even though they are energetically close in the Franck-Condon region, their overlap with the vibrational wave function of the ground state will be very different and Franck-Condon factors will favor the same Rydberg manifold in both experiments, i.e., the set of states created by exciting the same lone pair electron into one of the three $5 p$ and five $5 \mathrm{~d}$ Rydberg orbitals, respectively. Thus, it seems that the excited-state dynamics in the cation of 1,3-dibromopropane are of more general character than appreciated at first sight. We predict that one would observe the same dynamics in an experiment in which the REMPI process of the pump step involves an $(n, 5 s)$ Rydberg state as an intermediate state. In fact, a REMPI process involving any intermediate state that has a strong ionization correlation to the $\mathrm{D}_{3}$ state is expected to initiate the same cation dynamics.

\section{CONCLUSION}

We have conducted time-resolved ion photofragmentation experiments on the multichromophoric radical cation of 1,3dibromopropane, exploiting the interaction between the bromine atoms to probe the dynamics. By absorption of three photons of the pump pulse, the molecule is ionized through an intermediate $(n, 5 d)$ Rydberg state. The strong ionization correlation of the Rydberg state means that the cation is formed in the excited electronic state $\mathrm{D}_{3}$. The removal of an electron increases the interaction between the bromine atoms and initiates a coherent torsional vibration in the $\mathrm{D}_{3}$ state of the bromomethylene groups with a period of $0.7 \mathrm{ps}$. The $\mathrm{D}_{3}$ state decays by internal conversion to the $\mathrm{D}_{0}$ state in $1.6 \mathrm{ps}$.

Finally, we emphasize that the property that makes 1,3dibromopropane so well suited for investigations of structural and excited-state dynamics in the radical cation is the proximity of the two bromine atoms. The interaction between these two chromophores is crucial for probing the dynamics, demonstrating the applicability of a concept that we believe can be used in a wide range of systems. On this basis, we anticipate structural and excited-state cation dynamics to occur in multichromophoric molecules in which ionization enhances an interaction between two chromophores that was already present in the neutral molecule, a criteria that can be used for choosing potential candidates for future experiments.

\section{AUTHOR INFORMATION}

\section{Corresponding Author}

*E-mail: theis@kiku.dk.

\section{ACKNOWLEDGMENT}

We thank Dr. Steen Hammerum for enlightning discussions on radical cations of haloalkanes.

\section{REFERENCES}

(1) Ho, J.-W.; Chen, W.-K.; Cheng, P.-Y. J. Chem. Phys. 2009, $131,134308$.

(2) Baumert, T.; Röttgermann, C.; Rothenfusser, C.; Thalweiser, R.; Weiss, V.; Gerber, G. Phys. Rev. Lett. 1992, 69, 1512-1515.

(3) Cardoza, D.; Pearson, B. J.; Baertschy, M.; Weinacht, T. J. Photochem. Photobiol., A 2006, 180, 277-281.

(4) Cardoza, D.; Pearson, B. J.; Weinacht, T. J. Chem. Phys. 2007, 126, 084308.

(5) Geissler, D.; Pearson, B. J.; Weinacht, T. J. Chem. Phys. 2007, 127, 204305.

(6) Pearson, B. J.; Nichols, S. R.; Weinacht, T. J. Chem. Phys. 2007, 127, 131101.

(7) Loh, Z.-H.; Leone, S. R. J. Chem. Phys. 2008, 128, 204302.

(8) Yazawa, H.; Shioyama, T.; Hashimoto, H.; Kannari, F.; Itakura, R.; Yamanouchi, K. Appl. Phys. B: Lasers Opt. 2010, 98, 275-282.

(9) Rosenberg, M.; Minitti, M. P.; Rusteika, N.; Bisgaard, C. Z.; Deb, S.; Weber, P. M.; Sølling, T. I. J. Phys. Chem. A 2010, 114, 7021-7025.

(10) Plenge, J.; Wirsing, A.; Wagner-Drebenstedt, I.; Halfpap, I.; Kieling, B.; Wassermann, B.; Ruhl, E. Phys. Chem. Chem. Phys. 2011, 13, 8705-8714.

(11) Wagner, P. J. Acc. Chem. Res. 1983, 16, 461-467.

(12) Lakowicz, J. R. Principles of Fluorescence Spectroscopy, 3rd ed.; Klyuwer Academix/Plenum Publishers: Dordrecht, The Netherlands, 2006.

(13) Stolow, A.; Underwood, J. G. In Advances in Chemistry and Physics; Rice, S. A., Ed.; John Wiley \& Sons, Inc.: New York, 2008; Vol. 139; pp 497-584.

(14) Blanchet, V.; Zgierski, M. Z.; Seideman, T.; Stolow, A. Nature 1999, 401, 52-54.

(15) Schmitt, M.; Lochbrunner, S.; Shaffer, J. P.; Larsen, J. J.; Zgierski, M. Z.; Stolow, A. J. Chem. Phys. 2001, 114, 1206-1213.

(16) Blanchet, V.; Zgierski, M. Z.; Stolow, A. J. Chem. Phys. 2001, $114,1194-1205$.

(17) Cheng, W.; Kuthirummal, N.; Gosselin, J. L.; Sølling, T. I.; Weinkauf, R.; Weber, P. M. J. Phys. Chem. A 2005, 109, 1920-1925.

(18) Rusteika, N.; Møller, K. B.; Sølling, T. I. Chem. Phys. Lett. 2008, 461, 193-197.

(19) Rusteika, N.; Brogaard, R. Y.; Sølling, T. I.; Rudakov, F. M.; Weber, P. M. J. Phys. Chem. A 2009, 113, 40-43.

(20) Gosselin, J. L.; Minitti, M. P.; Rudakov, F. M.; Sølling, T. I.; Weber, P. M. J. Phys. Chem. A 2006, 110, 4251-4255.

(21) Frisch, M. J.; Trucks, G. W.; Schlegel, H. B.; Scuseria, G. E.; Robb, M. A.; Cheeseman, J. R.; Zakrzewski, V. G.; Montgomery, J. A., Jr.; Stratmann, R. E.; Burant, J. C.; Dapprich, S.; Millam, J. M.; Daniels, A. D.; Kudin, K. N. et al. Gaussian 03, Revision C.02; Gaussian, Inc.: Wallingford, CT, 2004. 
(22) Baboul, A. G.; Curtiss, L. A.; Redfern, P. C.; Raghavachari, K. J. Chem. Phys. 1999, 110, 7650-7657.

(23) Farup, P. E.; Stølevik, R. Acta Chem. Scand. A 1974, 28a, 680-692.

(24) Hasegawa, A.; Symons, M. C. R.; Shiotani, M. J. Chem. Soc., Perkin Trans. 2 1989, 657-665.

(25) Kötting, C.; Diau, E. W.-G.; Sølling, T. I.; Zewail, A. H. J. Phys. Chem. A 2002, 106, 7530-7546.

(26) NIST Chemistry WebBook; NIST Standard Reference Database Number 69; NIST: Gaithersburg, MD.

(27) Brogaard, R. Y.; Møller, K. B.; Sølling, T. I. J. Phys. Chem. A 2008, 112, 10481-10486. 